\title{
Suppression of shot noise in metallic diffusive conductors
}

\author{
C. W. J. Beenakker \\ Instituut-Lorentz, University of Leiden, P.O. Box 9506, 2900 RA Leiden, The Netherlands \\ M. Büttiker \\ IBM Thomas J. Watson Research Center, Yorktown Heights, New York 10598
}

(Received 3 April 1992)

\begin{abstract}
The shot-noise power in a disordered phase-coherent conductor, much longer than the mean free path but much shorter than an inelastic scattering length, is one-third of the classical value of a Poisson process. The reduction below the classical value is a consequence of noiseless open quantum channels. In conductors much longer than an inelastic length, shot noise is further suppressed due to counterbalancing voltage fluctuations which enforce charge neutrality.
\end{abstract}

Shot noise is the time-dependent fluctuation of the electrical current due to the discreteness of the charge of the carriers. In the zero-temperature limit, shot noise remains as the only source of electrical noise. For small applied voltages $V$, the shot-noise power $P$ is proportional to $V$, or equivalently to the time-averaged current $\bar{I}=G V$ (where $G$ is the conductance). In the absence of correlations among the carriers, the process of electrical conduction can be modeled by a Poisson process, for which

$$
P_{\text {Poisson }}=2 e|\bar{I}|=2 e|V| G .
$$

Correlations reduce the shot noise below the Poisson value (1). The noise power is therefore in general a new transport property, i.e., it contains information which is not present in the conductance.

The suppression of shot noise has been the subject of a large number of publications. We refer to the proceedings of a recent conference for references. ${ }^{1}$ The main interest thus far has been in the ballistic, resonant-tunneling and quantized Hall effect transport regimes. The case of a disordered phase-coherent conductor has received much less attention in this context. It is the purpose of the present paper to investigate the suppression of shot noise in the regime of diffusive quantum transport, and to study how loss of phase coherence by inelastic processes modifies the noise properties. One can distinguish two mechanisms which reduce the shot noise below Eq. (1). The first is the presence of open quantum channels, i.e., of eigenvalues of the transmission matrix product $\mathbf{t t}^{\dagger}$ which are of order unity in spite of the disorder. The second is the appearance of voltage fluctuations between regions separated by an inelastic length, which counterbalance the intrinsic current fluctuations of a phase-coherent region.

First, we consider the shot noise of a disordered conductor in the case of full phase coherence. We start from the general relation ${ }^{2}$

$$
\begin{aligned}
P & =2 e|V| \frac{e^{2}}{h} \operatorname{Tr} \mathbf{t t}^{\dagger}\left(1-\mathbf{t t}^{\dagger}\right) \\
& =2 e|V| \frac{e^{2}}{h} \sum_{n=1}^{N} T_{n}\left(1-T_{n}\right),
\end{aligned}
$$

between the current-noise spectral density $P$ and the eigenvalues $T_{n}(n=1,2, \ldots, N)$ of the transmission matrix product $\mathrm{tt}^{\dagger}$. The spectral density $P \equiv \overline{\Delta I^{2}} / \Delta \nu$ is defined as the statistical average (or time average) $\overline{\Delta I^{2}}$ of the square of the time-dependent current fluctuations $\Delta I(t) \equiv I(t)-\bar{I}$, divided by the frequency band width $\Delta \nu$ of the fluctuations. Equation (2) holds to first order in the applied voltage $V$, and in the zero-temperature, zerofrequency limit. It holds for arbitrary $N \times N$ transmission matrix $t$, generalizing results in Refs. $3-5$ for the singlechannel case $(N=1)$. We consider the case of a conductor of length $L$ much greater than the mean free path $l$ for elastic impurity scattering, but much smaller than the localization length $N l$. Equation (2) has not previously been evaluated in this regime of diffusive transport.

Our calculation applies a result from the randommatrix theory of quantum transport (see Ref. 6 for a recent review of this theory). We use the concept of a channel-dependent localization length $\zeta_{n}$, which is related to the transmission eigenvalue $T_{n}$ by $T_{n}=$ $\cosh ^{-2}\left(L / \zeta_{n}\right)$. The result we need is that the inverse localization length is uniformly distributed between 0 and $1 / \zeta_{\min } \simeq 1 / l$ for $l \ll L \ll N l .^{6}$ One can therefore write

$$
\begin{aligned}
\frac{\left\langle\sum_{n=1}^{N} f\left(T_{n}\right)\right\rangle}{\left\langle\sum_{n=1}^{N} T_{n}\right\rangle} & =\frac{\int_{0}^{L / \zeta_{\min }} d x f\left(\cosh ^{-2} x\right)}{\int_{0}^{L / \zeta_{\min }} d x \cosh ^{-2} x} \\
& =\int_{0}^{\infty} d x f\left(\cosh ^{-2} x\right),
\end{aligned}
$$

where $\langle\cdots\rangle$ indicates an average over an ensemble of impurity configurations. The function $f(T)$ is an arbitrary function of the transmission eigenvalue which vanishes for $T \ll 1$. In the second equality in Eq. (3) we have used that $L / \zeta_{\min } \simeq L / l \gg 1$ to replace the upper integration limit by $\infty$. With the help of Eq. (3) we can evaluate the ratio

$$
\frac{\left\langle\operatorname{Tr}\left(\mathbf{t} \mathbf{t}^{\dagger}\right)^{p}\right\rangle}{\left\langle\operatorname{Tr} \mathbf{t} \mathbf{t}^{\dagger}\right\rangle} \equiv C_{p}, \quad p=1,2, \ldots .
$$


Substitution of $f(T)=T^{p}$ into Eq. (3) gives the result

$$
C_{p}=\int_{0}^{\infty} d x \cosh ^{-2 p} x=\frac{\Gamma\left(\frac{1}{2}\right) \Gamma(p)}{2 \Gamma\left(p+\frac{1}{2}\right)} .
$$

Ratios of the form (4) have been studied previously by Pendry and co-workers. ${ }^{7}$ As far as we know, the result (5) is new. ${ }^{8}$

Since $C_{2}=\frac{2}{3}$, Eq. (2) implies that

$$
\langle P\rangle=2 e|V| \frac{e^{2}}{h}\left\langle\operatorname{Tr} \mathbf{t t}^{\dagger}\right\rangle\left(1-\frac{2}{3}\right)=\frac{2}{3} e|V|\langle G\rangle,
$$

where we have used the Landauer formula

$$
G=\frac{e^{2}}{h} \operatorname{Tr} \mathbf{t t}^{\dagger}
$$

The shot noise (6) is only one-third the value $\left\langle P_{\text {Poisson }}\right\rangle=$ $2 e|V|\langle G\rangle$ which would follow if the noise were a Poisson process ("full" shot noise). ${ }^{9}$ The reduction of shot noise in the diffusive transport regime which we have found is a quantum interference effect. It originates from the bimodal distribution of the transmission eigenvalues $T_{n}:$ A fraction $l / L$ of the eigenvalues is of order unity (open channels), the remainder being exponentially small (closed channels) ${ }^{7,10}$ In a semiclassical treatment, ${ }^{11}$ one would have instead $T_{n} \simeq l / L \ll 1$ for all $n$, so that [according to Eq. (2)] the shot noise $\langle P\rangle \simeq 2 e|V|\left(e^{2} / h\right)\left\langle\sum_{n} T_{n}\right\rangle=\left\langle P_{\text {Poisson }}\right\rangle$ takes on its full value.

Equations (1)-(7) are valid if the inelastic scattering length $l_{i}$ is much larger than the sample dimensions. Next we address the effect of phase and momentum randomizing events on shot noise in wires much longer than an inelastic scattering length. To accomplish this we extend earlier treatments which investigate the effect of inelastic events by attaching (one or more) voltage probes to the conductor. ${ }^{12}$ Consider a three-probe conductor. Contacts 1 and 2 are the current source and drain, and contact 3 is a voltage probe. The presence of a single voltage probe changes the two-terminal conductance $G \equiv G_{12,12}$ from Eq. (7) to ${ }^{12}$

$$
G=\frac{e^{2}}{h}\left(T_{21}+\frac{T_{23} T_{31}}{T_{31}+T_{32}}\right)
$$

The first term proportional to $T_{21} \equiv \operatorname{Tr} \mathrm{t}_{21} \mathrm{t}_{21}^{\dagger}$ represents coherent transmission from probe 1 to 2 . The second term represents incoherent transmission: Carriers which reach probe 3 (the voltage probe) are replaced by carriers from contact reservoir 3 with a phase which is uncorrelated to that of the incident carriers.

The current and voltage fluctuations in a three-probe conductor can be understood with the help of the equations $^{2}$

$$
I_{\alpha}=\frac{e}{h}\left(\left(N_{\alpha}-R_{\alpha \alpha}\right) \mu_{\alpha}-\sum_{\beta} T_{\alpha \beta} \mu_{\beta}\right)+\delta I_{\alpha} .
$$

Here $N_{\alpha}$ is the number of quantum channels in probe $\alpha, R_{\alpha \alpha}$ is the total probability for reflection back into probe $\alpha$, and $T_{\alpha \beta}$ is the total probability for transmission into probe $\alpha$ for carriers incident in probe $\beta$. For given (nonfluctuating) chemical potentials $\mu_{\alpha}$ of the contact reservoirs, the currents $I_{\alpha}$ in the probes have a timedependent fluctuating part $\delta I_{\alpha}$. In the zero-temperature limit these fluctuating currents have spectral weight ${ }^{2,13}$

$$
\begin{aligned}
\overline{\delta I_{\alpha} \delta I_{\beta}}=2 \Delta \nu \frac{e^{2}}{h} \int d E \sum_{\gamma, \delta(\delta \neq \gamma)} & f_{\gamma}\left(1-f_{\delta}\right) \\
& \times \operatorname{Tr}\left(\mathbf{s}_{\alpha \gamma}^{\dagger} \mathbf{s}_{\alpha \delta} \mathbf{s}_{\beta \delta}^{\dagger} \mathbf{s}_{\beta \gamma}\right),
\end{aligned}
$$

where $f_{\gamma}=\theta\left(\mu_{\gamma}-E\right)$ is the zero-temperature Fermi function, and $s_{\alpha \beta}$ are the scattering matrices which connect the outgoing current amplitudes at probe $\alpha$ with the incoming current amplitudes at probe $\beta$. The scattering matrices determine the total reflection and transmission probabilities: $R_{\alpha \alpha}=\operatorname{Tr} \mathbf{s}_{\alpha \alpha} \mathbf{s}_{\alpha \alpha}^{\dagger}, T_{\alpha \beta}=\operatorname{Tr} \mathbf{s}_{\alpha \beta} \mathbf{s}_{\alpha \beta}^{\dagger}$. We also denote $\mathbf{s}_{\alpha \alpha} \equiv \mathbf{r}_{\alpha \alpha}, \mathbf{s}_{\alpha \beta} \equiv \mathbf{t}_{\alpha \beta}$.

Here we investigate Eqs. (9) and (10) in the limit where transmission through the sample is completely incoherent. In this limit coherent transmission from probe 1 to probe 2 is absent, i.e., we have $\mathbf{s}_{21}=\mathbf{s}_{12}=0$ and consequently $T_{21}=T_{12}=0$. We then find from Eq. (10) for the mean square currents (to first order in the chemical potential differences)

$$
\begin{aligned}
& \overline{\delta I_{1}^{2}}=2 \Delta \nu \frac{e^{2}}{h}\left|\mu_{1}-\mu_{3}\right| \operatorname{Tr} \mathbf{r}_{11} \mathbf{r}_{11}^{\dagger} \mathbf{t}_{13} \mathrm{t}_{13}^{\dagger}, \\
& \overline{\delta I_{2}^{2}}=2 \Delta \nu \frac{e^{2}}{h}\left|\mu_{3}-\mu_{2}\right| \operatorname{Tr} \mathbf{r}_{22} \mathbf{r}_{22}^{\dagger} \mathrm{t}_{23} \mathbf{t}_{23}^{\dagger}, \\
& \overline{\delta I_{3}^{2}}=\overline{\delta I_{1}^{2}}+\overline{\delta I_{2}^{2}},
\end{aligned}
$$

and for the correlations of the fluctuations

$$
\overline{\delta I_{1} \delta I_{2}}=0, \quad \overline{\delta I_{1} \delta I_{3}}=-\overline{\delta I_{1}^{2}}, \overline{\delta I_{2} \delta I_{3}}=-\overline{\delta I_{2}^{2}} .
$$

For given $\mu_{3}$, i.e., in the absence of voltage fluctuations, the correlation of the current fluctuations at probes 1 and 2 vanishes as a consequence of the inelastic scattering.

The average value of the chemical potential $\mu_{3}$ at the voltage probe is determined from the condition that an ideal voltmeter has an infinite internal impedance, and that consequently the average current $\vec{I}_{3}$ at the voltage probe must vanish. The time-dependent fluctuations in $\mu_{3}$ can be determined from the condition $\Delta I_{3}=0$, i.e., by requiring that at the voltage probe it is not just the average current which vanishes but the total current. In principle, one can consider a voltmeter with a finite impedance, for which $\Delta I_{3} \neq 0$. Note that the lowfrequency theory given here conserves the total current. Indeed, the sum of all mean square currents and all correlations [i.e., Eqs. (11)-(13) plus twice Eqs. (14)] is zero, as required by $\delta I_{1}+\delta I_{2}+\delta I_{3}=0$. Similarly, the sum of the fluctuations of the total currents of Eq. (9) vanishes, $\Delta I_{1}+\Delta I_{2}+\Delta I_{3}=0$. A nonzero $\Delta I_{3}$ therefore leads to a temporary loss or temporary accumulation of charge. At small frequencies a metal will maintain charge neutrality, and hence the model of an ideal voltmeter (infinite impedance, $\Delta I_{3}=0$ ) considered here is appropriate.

We therefore seek a solution of Eq. (9) for given $\mu_{1}$ 
and $\mu_{2}$ under the condition that $I_{3}=0$. Without loss of generality we can take $\mu_{2}=0$, and find

$\mu_{3}=\frac{T_{31}}{T_{13}+T_{23}} \mu_{1}-\frac{1}{T_{13}+T_{23}} \delta I_{3} \equiv \bar{\mu}_{3}+\delta \mu_{3}$,

where we have used $N_{3}-R_{33}=T_{13}+T_{23}$. The first term $\bar{\mu}_{3}$ is the average value of the voltage at probe 3 needed to keep the average current $\bar{I}_{3}$ zero. The second term $\delta \mu_{3}$ is the voltage fluctuation in response to the intrinsic current fluctuations. According to Eq. (9) the current at probe 1 now fluctuates with amplitude $\Delta I_{1}=\delta I_{1}-T_{13} \delta \mu_{3}$. Using Eq. (15), and substituting $\delta I_{3}=-\delta I_{1}-\delta I_{2}$, we obtain

$$
\Delta I_{1}=\frac{T_{23} \delta I_{1}-T_{13} \delta I_{2}}{T_{13}+T_{23}} .
$$

A similar calculation for the fluctuation amplitude of the current at probe 2 shows that $\Delta I_{2}=-\Delta I_{1}$, as required by current conservation (since $I_{3}=0$ ). Therefore, we find

$$
\begin{aligned}
& \overline{\Delta I_{1}^{2}}=\overline{\Delta I_{2}^{2}}=-\overline{\Delta I_{1} \Delta I_{2}}, \\
& \overline{\Delta I_{1}^{2}}=\frac{T_{23}^{2} \overline{\delta I_{1}^{2}}+T_{13}^{2} \overline{\delta I_{2}^{2}}-2 T_{13} T_{23} \overline{\delta I_{1} \delta I_{2}}}{\left(T_{13}+T_{23}\right)^{2}} .
\end{aligned}
$$

The fluctuations at probes 1 and 2 of the total current are now completely correlated. This correlation is a consequence not of phase coherence but of current conservation in a conductor which maintains charge neutrality. Equation (18) can be evaluated with the help of the fluctuation spectra (10). The Fermi functions which appear in Eq. (10) refer to the average chemical potential: $f_{\gamma}=\theta\left(\bar{\mu}_{\gamma}-E\right)$.

Let us now consider the limiting case of completely incoherent transmission. Then, according to Eq. (14), the correlation $\overline{\delta I_{1} \delta I_{2}}$ of the intrinsic current fluctuations vanishes, while the mean squared current fluctuations are given by Eqs. (11) and (12). Inserting these expressions into Eq. (18), and taking into account that $\mu_{1}-\bar{\mu}_{3}=$ $\left(\mu_{1}-\mu_{2}\right) T_{32} /\left(T_{13}+T_{23}\right)$ and $\bar{\mu}_{3}-\mu_{2}=\left(\mu_{1}-\mu_{2}\right) T_{31} /\left(T_{13}+\right.$ $\left.T_{23}\right)$, we find for the noise spectral density $P \equiv \overline{\Delta I_{1}^{2}} / \Delta \nu$ the expression

$$
P=2 e|V| \frac{e^{2}}{h} \frac{T_{23}^{3} \operatorname{Tr}\left(\mathbf{r}_{11} \mathbf{r}_{11}^{\dagger} \mathbf{t}_{13} \mathrm{t}_{13}^{\dagger}\right)+T_{13}^{3} \operatorname{Tr}\left(\mathbf{r}_{22} \mathbf{r}_{22}^{\dagger} \mathrm{t}_{23} \mathrm{t}_{23}^{\dagger}\right)}{\left(T_{13}+T_{23}\right)^{3}},
$$

with $\mu_{1}-\mu_{2} \equiv \mathrm{eV}$. Here we have used that $T_{12}=T_{21}=0$ implies $T_{32}=T_{23}$ and $T_{31}=T_{13}$, because of unitarity of the scattering matrix.

Equation (19) can be written in a more transparent way by defining the resistances $R_{1} \equiv h / 2 e^{2} T_{13}$, $R_{2}=h / 2 e^{2} T_{23}, R \equiv R_{1}+R_{2}$, and the noise powers $P_{1} \equiv 2 e|V|\left(R_{1} / R\right)\left(e^{2} / h\right) \operatorname{Tr} \mathrm{t}_{13} \mathrm{t}_{13}^{\dagger}\left(1-\mathrm{t}_{13} \mathrm{t}_{13}^{\dagger}\right), P_{2} \equiv$ $2 e|V|\left(R_{2} / R\right)\left(e^{2} / h\right) \operatorname{Tr} t_{23} t_{23}^{\dagger}\left(1-t_{23} t_{23}^{\dagger}\right)$. Equation (19) is then equivalent to the equation

$$
R^{2} P=R_{1}^{2} P_{1}+R_{2}^{2} P_{2} .
$$

This addition rule has a simple interpretation. The lefthand side $R^{2} P=\overline{\Delta V_{12}^{2}} / \Delta \nu$ equals the spectral density of voltage fluctuations of the conductor, which one would measure by connecting a voltmeter to contacts 1 and 2 . The right-hand side $R_{1}^{2} P_{1}+R_{2}^{2} P_{2}=\overline{\Delta V_{13}^{2}} / \Delta \nu+\overline{\Delta V_{23}^{2}} / \Delta \nu$ is the sum of the spectral densities of the voltage fluctuations measured between contacts 1 and 3 and between contacts 2 and 3 . The addition rule (20) thus states that the voltage fluctuations $\Delta V_{13}$ and $\Delta V_{23}$ are statistically independent, so that the variances add. This is a known result for classical resistors in series, in which the shot noise is Poissonian. ${ }^{14}$ The present analysis extends this addition rule to the quantum transport regime, where open channels lead to sub-Poissonian shot noise.

We can similarly model a wire of length $L$ much longer than the inelastic scattering length $l_{i}$ by a series of phasecoherent segments of length $l_{i}$, separated by phase and momentum randomizing voltage probes. If each segment $s$ individually has resistance $R_{s}$ and noise power $P_{s}$, then the noise power $P$ of the whole wire (with resistance $R=$ $\sum_{s} R_{s}$ ) satisfies the addition rule $R^{2} P=\sum_{s} R_{s}^{2} P_{s}$. In the case that the phase-coherent segments have roughly equal transport properties one has $R_{s} \approx R\left(l_{i} / L\right)$, so that $P$ has become smaller than the noise power $P_{s}$ of an individual segment by a factor of order $l_{i} / L .^{11}$

We write the ensemble average of Eq. (19) in the form

$$
\begin{aligned}
& \langle P\rangle=2 e|V| \frac{e^{2}}{h}\left\langle F\left(\sum_{n} T_{n}(13), \sum_{n} T_{n}(23), \sum_{n} T_{n}^{2}(13), \sum_{n} T_{n}^{2}(23)\right)\right\rangle, \\
& F\left(x_{1}, x_{2}, y_{1}, y_{2}\right) \equiv \frac{x_{2}^{3}\left(x_{1}-y_{1}\right)+x_{1}^{3}\left(x_{2}-y_{2}\right)}{\left(x_{1}+x_{2}\right)^{3}}
\end{aligned}
$$

where $T_{n}(13)$ and $T_{n}(23)(n=1,2, \ldots, N)$ are the eigenvalues of $\mathbf{t}_{13} \mathbf{t}_{13}^{\dagger}$ and $\mathbf{t}_{23} \mathbf{t}_{23}^{\dagger}$, respectively. A complication, arises because $F$ is a nonlinear function of its arguments, so that in general we cannot replace the ensemble average of $F$ by the function of the ensemble-averaged arguments. This is only justified if the fluctuations of the arguments around their average are small. Now we note that, as a result of the bimodal distribution of the transmission eigenvalues, both the sums $\sum_{n} T_{n}$ and $\sum_{n} T_{n}^{2}$ are of order $N l / L$, being the number of open channels of the con- ductor. It is a general result of random-matrix theory ${ }^{6,10}$ that fluctuations over the ensemble in the number of open channels are of order unity, and hence that the fluctuations in the quantities $\sum_{n} T_{n}$ and $\sum_{n} T_{n}^{2}$ are a factor of order $L / N l$ smaller than the average. ${ }^{15}$ In the limit $L \ll N l$ of a conductor small compared to the localization length, we can, therefore, replace the ensemble average of $F$ by the function of the ensemble averaged arguments. Using the result $\left\langle\sum_{n} T_{n}^{2}\right\rangle=\frac{2}{3}\left\langle\sum_{n} T_{n}\right\rangle$ we find 


$$
\langle P\rangle=\frac{1}{3}\left\langle P_{\text {Poisson }}\right\rangle \frac{\left\langle T_{23}\right\rangle^{2}+\left\langle T_{13}\right\rangle^{2}}{\left(\left\langle T_{13}\right\rangle+\left\langle T_{23}\right\rangle\right)^{2}}
$$

Equation (23) describes two effects which act to reduce shot noise below the Poisson value: inelastic scattering and the absence of noise in open quantum channels. If $\left\langle T_{23}\right\rangle=\left\langle T_{13}\right\rangle$, then Eq. (23) predicts that the shot-noise power is only one-sixth of the Poisson value, as a result of a factor of 3 reduction from open channels and a factor of 2 reduction from inelastic scattering.

We conclude by noting that, apart from being of intrinsic interest, the suppression of shot noise ccnsidered here has important applications: The fluctuations of the (electric) source used to pump a laser also determine the noise properties of the emitted light. Large resistors in series with the source are used to suppress shot noise and to achieve a nonchaotic light source. ${ }^{16}$ The suppression of shot noise is possibly also at the root of observations of Coulomb-blockade effects in single normal junctions.

Research at the University of Leiden was supported financially by the "Nederlandse organisatie voor Wetenschappelijk Onderzoek" (NWO) via the "Stichting voor Fundamenteel Onderzoek der Materie" (FOM).
${ }^{1}$ R. Landauer and Th. Martin, Physica 175, 167 (1991); M. Büttiker, ibid. 175, 199 (1991).

${ }^{2}$ M. Büttiker, Phys. Rev. Lett. 65, 2901 (1990).

${ }^{3}$ V. A. Khlus, Zh. Eksp. Teor. Fiz. 93, 2179 (1987) [Sov. Phys. JETP 66, 1243 (1987)].

${ }^{4}$ G. B. Lesovik, Pis'ma Zh. Eksp. Teor. Fiz. 49, 513 (1989) [JETP Lett. 49, 592 (1989)].

${ }^{5}$ B. Yurke and G. P. Kochanski, Phys. Rev. B 41, 8184 (1990).

${ }^{6}$ A. D. Stone, P. A. Mello, K. A. Muttalib, and J.-L. Pichard, in Mesoscopic Phenomena in Solids, edited by B.

L. Al'tshuler, P. A. Lee, and R. A. Webb (North-Holland, Amsterdam, 1991).

${ }^{7}$ J. B. Pendry, A. MacKinnon, and A. B. Pretre, Physica A 168, 400 (1990); J. B. Pendry, A. MacKinnon, and P. J. Roberts, Proc. R. Soc. London Ser. A 437, 67 (1992); J. B. Pendry (private communication) has compared our analytical formula (5) for $C_{p}$ with their numerical simulations based on a tight-binding model of a disordered twoand three-dimensional system. The numerical results in the metallic (delocalized) regime are quite close the analytical ones [e.g., $C_{2} \approx 0.653, C_{3} \approx 0.514$ (numerically, for a cubic lattice), versus $C_{2}=\frac{2}{3} \approx 0.667, C_{3}=\frac{8}{15} \approx 0.533$ (analytically)].

${ }^{8}$ M. J. M. de Jong has pointed out to us that the coefficient $C_{2}=\frac{2}{3}$ can also be deduced from the moment equations derived by P. A. Mello and A. D. Stone, Phys. Rev. B 44,
3559 (1991).

${ }^{9}$ We note that the one-third reduction factor in the shot noise due to open quantum channels is independent of the universality class of the ensemble of scattering matrices (the $\beta$ parameter of random-matrix theory). In particular, it does not depend on whether time-reversal symmetry is broken or not by a (weak) magnetic field. The reason for this independence is the insensitivity of the density of transmission eigenvalues to the symmetry of the system, as demonstrated explicitly by P. A. Mello and J.-L. Pichard, Phys. Rev. B 40, 5276 (1989).

${ }^{10}$ Y. Imry, Europhys. Lett. 1, 249 (1986).

${ }^{11}$ C. W. J. Beenakker and H. van Houten, Phys. Rev. B 43, 12066 (1991).

${ }^{12}$ M. Büttiker, Phys. Rev. B 33, 3559 (1986); IBM J. Res. Dev. 32, 63 (1988).

${ }^{13}$ For additional closely related discussions see M. Büttiker, Phys. Rev. Lett. 68, 843 (1992); Th. Martin and R. Landauer, Phys. Rev. B 45, 1742 (1992).

${ }^{14}$ A. van der Ziel, Noise in Solid State Devices and Circuits (Wiley, New York, 1986).

${ }^{15}$ This also implies that the mesoscopic, sample-to-sample fluctuations in the noise power $P$ have a root-mean-square value $\operatorname{rms} P \simeq e|V|\left(e^{2} / h\right)$, up to a numerical coefficient of order unity.

${ }^{16} \mathrm{~S}$. Machida, Y. Yamamoto, and Y. Itaya, Phys. Rev. Lett. 58, 1000 (1987). 